\title{
Lithium iron phosphate intelligent SOC prediction for efficient electric vehicle
}

\begin{abstract}
This paper presents modelling techniques for Lithium Iron Phosphate (LiFePO4) battery in an electric vehicle. Artificial intelligence techniques namely multi-layered perceptron neural network (MLPNN) and Elman recurrent neural network are devised to estimate the energy remained in the battery bank which referred to state of charge (SOC). The New European Driving Cycle (NEDC) test data is used to excite the cells in driving cycle-based conditions under varied temperature range $[0-55]^{\circ} \mathrm{C}$. Accurate SOC prediction is a key function for satisfactory implementation of Battery Supervisory System (BSS). It is demonstrated that artificial intelligence methods can be effectively used with highly accurate results. The accuracy of the modeling results is demonstrated through validation and correlation tests.
\end{abstract}

Keyword: Elman recurrent neural network and battery supervisory system (BSS); Lithium iron phosphate; Multi-layered perceptron neural network (MLPNN); State of charge (SOC) 